\title{
Medicaid managed care: Issues for beneficiaries with disabilities
}

\author{
Jean P. Hall, Ph.D. ${ }^{\text {a,d,* }}$, Noelle K. Kurth, M.S. ${ }^{a}$, Shawna L.C. Chapman, Ph.D. ${ }^{\text {, }}$, and \\ Theresa I. Shireman, Ph.D. ${ }^{\circ}$ \\ ${ }^{a}$ Institute for Health and Disability Policy Studies, University of Kansas, 1122 West Campus Rd., Room 517, Lawrence, KS 66045, USA \\ ${ }^{\mathrm{b}}$ Duke University School of Medicine Department of Psychiatry and Behavioral Sciences, Box 3903, Durham, NC 27710, USA \\ ${ }^{\mathrm{c}}$ Department of Preventive Medicine and Public Health, University of Kansas Medical Center, 3901 Rainbow Blvd, Mailstop 1008, 4030 Robinson, \\ Kansas City, KS 66160, USA \\ ${ }^{\mathrm{d}}$ Department of Health Policy and Management, University of Kansas Medical Center, 3901 Rainbow Boulevard, Mailstop 3044,5001 Student Center, \\ Kansas City, KS 66160, USA
}

\begin{abstract}
Background: States are increasingly turning to managed care arrangements to control costs in their Medicaid programs. Historically, such arrangements have excluded people with disabilities who use long-term services and supports (LTSS) due to their complex needs. Now, however, some states are also moving this population to managed care. Little is known about the experiences of people with disabilities during and after this transition.

Objective: To document experiences of Medicaid enrollees with disabilities using long-term services and supports during transition to Medicaid managed care in Kansas.

Methods: During the spring of 2013, 105 Kansans with disabilities using Medicaid long-term services and supports (LTSS) were surveyed via telephone or in-person as they transitioned to managed care. Qualitative data analysis of survey responses was conducted to learn more about the issues encountered by people with disabilities under Medicaid managed care.

Results: Respondents encountered numerous disability-related difficulties, particularly with transportation, durable medical equipment, care coordination, communication, increased out of pocket costs, and access to care.

Conclusions: As more states move people with disabilities to Medicaid managed care, it is critically important to address these identified issues for a population that often experiences substantial health disparities and a smaller margin of health. It is hoped that the early experiences reported here can inform policy-makers in other states as they contemplate and design similar programs. (C) 2015 Elsevier Inc. All rights reserved.
\end{abstract}

Keywords: Disabilities; Medicaid; Managed care; Long-term services and supports (LTSS)

Over the last few years, more and more states have moved at least parts of their Medicaid populations to managed care. ${ }^{1-3}$ Due to their complex health care needs, people with disabilities were often left out of such arrangements. However, states are increasingly experimenting with adding at least some groups with greater health care needs to managed care. $^{4-7}$ Many Medicaid beneficiaries with disabilities use long-term services and supports (LTSS), usually through

External funding information: Portions of data collection for this project were conducted under a grant from the U.S. Department of Education, National Institute on Disability and Rehabilitation Research (NIDRR) grant number H133G100082. However, the contents do not necessarily reflect the policy of the Department of Education, and readers should not assume endorsement by the Federal Government.

Findings from the study were presented at the AcademyHealth Annual Research Meeting in San Diego, CA on June 8, 2014.

The authors have no conflicts of interest to declare.

* Corresponding author. Tel.: +1 785864 7083; fax: +1 7858647799 .

E-mail address: jhall@ku.edu (J.P. Hall). home and community-based service (HCBS) waivers, for assistance with activities of daily living. While these services are less medical in nature, they are nonetheless critical to the health and function of many people with disabilities. Because people with disabilities, particularly those using LTSS, are an expensive population for state Medicaid programs, ${ }^{8}$ the interest by states in using managed care to potentially cut costs is not surprising. In 2012, 16 states placed at least some beneficiaries using LTSS into Medicaid managed care, with only seven doing so statewide for all LTSS, for a total of about 390,000 beneficiaries nationally. In 2014, 26 states are projected to have managed care programs in place for Medicaid LTSS, including HCBS, for a total of more than 1.8 million people. ${ }^{7,9}$

The state of Kansas implemented statewide Medicaid managed care, known as KanCare, in January 2013. Beneficiaries were auto-assigned to one of three for-profit managed care organizations (MCOs), with the option to 
change to one of the others if desired. ${ }^{10,11}$ KanCare includes the adult disabled population and all HCBS waiver participants. Previously, the adult disabled population had been in a fee-for-service program with access to all Medicaid providers and with case management of their LTSS provided by local community-based organizations.

People with disabilities are particularly sensitive to small changes in access to care and have unique needs that may differ substantially from those of other Medicaid beneficiaries. ${ }^{4,7,12}$ It is therefore extremely important to document their experiences, and needed improvements in their services, as more and more people with disabilities are moved to managed care. ${ }^{13}$ Other studies documenting the use of managed care for Medicaid LTSS have been conducted in the past few years. ${ }^{5,10,14,15}$ None of these studies, however, gathered data directly from the Medicaid beneficiaries themselves, but rather based findings on policy reviews and interviews with providers, advocates, legislators, and Medicaid agency staff. To fill this critical gap in the literature, 105 adults with a variety of disabilities who use LTSS were surveyed during the transition to the KanCare program. The aim was to assess consumer satisfaction at approximately six months into the new program and to identify early challenges and gaps in care occurring under this new delivery system. While a majority of study participants reported being satisfied with KanCare, some disability-related concerns emerged and are documented here. These issues must be addressed by managed care organizations to serve this population better in the future.

\section{Methods}

This study utilized a survey administered in person or via telephone with KanCare participants receiving LTSS through a Medicaid HCBS waiver or the Medicaid Buy-In program in Kansas. The survey was conducted between April and August 2013, subsequent to implementation of KanCare, to document early experiences for this population.

\section{Sample}

The sampling frame for this study included participants from a prior survey of Kansans with disabilities ${ }^{16}$ aged 18-64 $(n=69)$ and new participants $(n=36)$ recruited via flyers posted at Centers for Independent Living (CILs) across the state. The survey was administered by telephone $(n=69)$ or in-person at CILs $(n=36)$ by two researchers following scripted administration protocols developed for the study. All potential participants were given the option of being entered in a drawing for a $\$ 100$ gift card regardless of survey completion. Informed consent was obtained verbally and responses deidentified. The University of Kansas Institutional Review Board approved this study.

\section{Survey instrument(s)}

The survey instrument contained demographic items and health care access questions. Respondents were asked if they were able to access certain types of health care (medical care from a doctor, services from a mental health provider or counselor, and prescription drugs) when needed "since KanCare began." Items also included open-ended probes for those who indicated they had access problems. Additional questions, some open-ended, queried participants about their general experiences since implementation of managed care, particularly regarding communication, the managed care organizations, and satisfaction with the KanCare plans.

\section{Analyses}

Descriptive statistics were utilized to characterize the sample (Table 1). A researcher trained in qualitative methods who did not administer surveys analyzed the open-ended response data using conventional content analysis, a methodology that requires researchers to immerse themselves in data and allows codes, categories and themes to emerge. ${ }^{17,18}$ The researcher initially read data, identified pertinent areas of text, pile sorted areas into like categories and used categories to construct codes. Data were reread and coded and final coded text was used to construct themes. ${ }^{18,19}$ After discussing preliminary themes with the other researchers, including those who administered the survey, and developing consensus on topics, she again reviewed and sorted the responses into identified topics and confirmed themes. Researchers met again to discuss and concur with final themes. This methodology is commonly used for analysis of data in similar studies. ${ }^{20-25}$

\section{Results}

The study sample demographics are included in Table 1; the sample was drawn from approximately 15,000 people with disabilities enrolled in one of the three adult, non-elderly waiver programs in Kansas (physical disability, traumatic brain injury, and intellectual/developmental disability). ${ }^{26}$ A majority of respondents $-64 \%$ - reported being satisfied or very satisfied overall with KanCare. Overall, 9.5\% reported that their benefits had improved while $13.3 \%$ reported their benefits had gotten worse after implementation of managed care (Table 1). Compared to previous surveys with this population, ${ }^{16}$ respondents were more likely to report fair or poor health and to have difficulties obtaining certain types of medical care post-KanCare implementation, particularly mental health providers and prescription drugs. Qualitative analysis of open-ended items yielded five distinct categories of respondent comments regarding their experiences with KanCare: 1) provider networks, 2) limitations in covered benefits, 3) transportation, 4) communication and 5) care coordination. These 
Table 1

Sample demographics and selected survey items $(n=105)$

\begin{tabular}{|c|c|}
\hline Item & $\%$ \\
\hline \multicolumn{2}{|l|}{ Demographics } \\
\hline Mean age (SD, range) & $50.8(10.5,27-82)$ \\
\hline Gender, female & 52.4 \\
\hline Race, white & 80.0 \\
\hline Ethnicity, Hispanic & 2.9 \\
\hline \multicolumn{2}{|l|}{ Disability type } \\
\hline Physical $^{\mathrm{a}}$ & 40.0 \\
\hline Intellectual $^{\mathrm{b}}$ & 24.8 \\
\hline Chronic illness $^{c}$ & 22.9 \\
\hline SPMI $^{\mathrm{d}}$ & 7.6 \\
\hline Sensory & 3.8 \\
\hline Undisclosed & 1.0 \\
\hline \multicolumn{2}{|l|}{ Location } \\
\hline Frontier/rural $^{\mathrm{f}}$ & 32.4 \\
\hline Semi-urban/urban ${ }^{\mathrm{g}}$ & 32.4 \\
\hline Metropolitan ${ }^{\mathrm{h}}$ & 35.2 \\
\hline Report fair or poor health & 57.2 \\
\hline \multicolumn{2}{|l|}{ Access to care \& satisfaction measures } \\
\hline Unable to access needed medical services & 24.8 \\
\hline No access problems identified & 72.3 \\
\hline Don't know & 2.9 \\
\hline Unable to access needed waiver services & 16.2 \\
\hline No access problems identified & 72.4 \\
\hline Don't know & 11.4 \\
\hline \multicolumn{2}{|l|}{ Overall satisfaction with $\mathrm{MCO}$} \\
\hline Satisfied/very satisfied & 63.8 \\
\hline Neither & 16.2 \\
\hline Dissatisfied/very dissatisfied & 10.5 \\
\hline Don’t know & 9.5 \\
\hline \multicolumn{2}{|c|}{$\begin{array}{l}\text { Report change in Medicaid medical benefits after managed care } \\
\text { implementation }\end{array}$} \\
\hline Improved & 9.5 \\
\hline Gotten worse & 13.3 \\
\hline Stayed the same & 64.8 \\
\hline Don't know & 12.4 \\
\hline \multicolumn{2}{|c|}{$\begin{array}{l}\text { Respondents with comments pertaining to five themes identified via } \\
\text { qualitative analysis }\end{array}$} \\
\hline Limitations in covered benefits & 21.9 \\
\hline Provider networks & 13.3 \\
\hline Communication & 12.4 \\
\hline Care coordination & 11.4 \\
\hline Transportation & 10.5 \\
\hline Reported at least one problem theme & 45.7 \\
\hline Reported two or more problem themes & 18.1 \\
\hline
\end{tabular}

${ }^{a}$ e.g., paraplegia, spina bifida, amputations and traumatic brain injury.

b e.g., Down syndrome, phenylketonuria, and autism.

c e.g., end stage renal disease, lupus, epilepsy, and cystic fibrosis.

d e.g., schizophrenia, bipolar disorder, and depression.

e e.g., hearing and visual impairments, deafness and blindness.

f $\leqslant 40$ people/sq mile.

g 41-149 people/sq mile.

$\mathrm{h} \geqslant 150$ people/sq mile.

themes are provided with representative quotations in Table 2 and described below.

\section{Provider networks}

When asked if they were unable to access needed medical services since KanCare began, $24.8 \%$ responded yes (Table 1). One respondent, living on the border to another state, reported that the specialists she had seen for years in the adjacent state were unwilling to accept KanCare. Similarly, others reported being unable to see specialists they needed and were able to see in the past such as neurologists, urologists, dentists, optometrists, and (most commonly) mental health therapists or counselors. Having a limited pool of providers available was also cited as challenging, especially in the more rural areas of the state. One consumer said, "Speech therapy is a two-and-a-half month wait because there's only one provider for my MCO."

\section{Limitations in covered benefits}

Respondents particularly noted problems accessing medications post-KanCare implementation. Medications that were difficult to obtain included those for pain and incontinence, as well as over-the-counter medicines that were previously covered. Respondents now had to pay for these medications out of pocket or simply stop taking them: "I can't get [medication name], so I'm just not taking it. I need it, though." Additional coverage limitations were reported, including one individual who explained that catheters were covered, but not the gel needed to insert them saying, "It makes no logical sense." Another respondent reported, "I have extreme neuropathy in my hands and have to use a certain type of [glucose] meter because of my hand weakness. My MCO won't cover that type; I may have to stop testing." Other limitations related to individuals' disabilities were also mentioned, "I use a wheelchair and get pressure sores on my feet. My doctor says I need a shoe insert to stop them, but it's not covered. So, I wait until it gets more major and then go to the doctor or hospital repeatedly ..." And, "I had to pay $\$ 700$ to get a joystick on my new wheelchair; they [MCO] won't cover it even though I'm a quad [riplegic]." Finally, 16.2\% of respondents reported they had been unable to access all of the waiver services they did before KanCare (Table 1).

\section{Transportation}

While the survey did not ask questions specifically related to transportation, respondents independently cited transportation as an issue. Prior to KanCare, most nonemergency medical transportation was provided by locally-operated and familiar entities. After KanCare implementation, several respondents had difficulty obtaining accessible, medically necessary transportation. A woman who uses a power wheelchair reported instances of being sent an inaccessible car and being transferred incorrectly, resulting in a lack of safety and consequent injury. Similarly, respondents reported that their personal care attendants, on whom they rely for assistance with transferring and other tasks, were not allowed to be paid to accompany them to medical appointments. Others reported that the notice required to obtain transportation was a barrier: "My doctor's office changed my appointment time, but 
Table 2

Consumer experiences with managed care

\begin{tabular}{ll}
$n^{\mathrm{a}}$ & Category/theme \\
\hline 14 & Provider networks
\end{tabular}

$14 \quad$ Provider networks

Representative quotes
"None of the providers I have seen for years in Jackson County Missouri takes KanCare."
"I can't see my neurologist."
"I can't find a mental health therapist who accepts KanCare."
"I am finding no dentists in western Kansas who will accept KanCare and our optician won't participate because of the hassle."
"Speech therapy is a two and a half month wait because there's only one provider for my MCO."

23 Limitations in covered benefits

"I can get all except pain relief, it is over the counter and they won't pay for that."

"Can't get [medication name], so I'm just not taking it. I Need it though."

"MCO does not cover my incontinence medicine, so now I have to pay out of pocket."

"I have extreme neuropathy in my hands and have to use a certain type of meter because of the hand weakness. My MCO won't cover that type; I may have to stop testing my blood."

"They [MCO] cover catheters, but the gel for catheter insertion is no longer covered. It is only \$5-6 a tube, but it makes no logical sense."

"I use a wheelchair and get pressure sores on my feet. My doctor says I need a shoe insert to stop the sores, but it's not covered. So, I wait until it is more major and go to the doctor or hospital repeatedly for them to fix the aftermath."

"I had to pay \$700 to get a joystick on my new wheelchair; they [MCO] won't cover it even though I'm a quad [riplegic]."

"I need a new cane or walker and a shower chair. I can't get them even though I have a doctor's note."

11 Transportation

"The MCO sent an inaccessible car when they did actually send one."

"The transportation people don't transfer me correctly and I've gotten hurt."

"If my PCA [personal care attendant] doesn't go, I'm not getting strapped in correctly and it's unsafe."

"My PCA can't get paid to go along with me."

"She [the driver] can't properly transport me without just about killing me-it's not safe."

"Once a cab driver showed up with his dog with him. I am allergic to dogs."

"Someone comes from out-of-state-no local provider-and I don't know these people and they don't treat me well or properly. Before, you knew the person, they were local and usually the same person each time."

"I have transportation up to appointments in Kansas City and they [MCO] provide it. But, sometimes I have two appointments and they won't wait. So, I have to get a hotel and they come back the next day. Very expensive!"

"My doctor's office changed my appointment time, but the MCO won't change the pickup time because it is not three days' notice."

"Three days notice is hard to give sometimes."

13 Communication

"The 800 number tells you to go online, but they don't realize that not everyone has internet access."

"The MCO said I could get a cell phone with $250 \mathrm{~min}$ for free, but as a quad, I can't use their phone because the buttons are too small. I Asked if I could use my existing phone (the same cell company), but was told no, they won't allow it."

"Sometimes when I call the MCO the person says they can't understand me [due to speech impairment]. It's rude. They aren't taking the time to try and understand me. I mean, I'm talking on the phone with you and you can understand me."

"When I call the MCO toll-free line, the message says, 'if you are mentally ill or not competent to handle these procedures [of the automated system], perhaps you should talk to a person.' That's offensive, to have a computer tell me I am incompetent. And, you can't actually get a person. I am hesitant to call now."

"Get so much mail all the time ... it's hard to remember which is which and what to do with it. I don't understand all of it."

"I don't like dealing with all the confusing paperwork and everything coming in the mail."

12 Care coordination

"I had more direct contact with my case manager before [KanCare]. Now, I have to call a number, leave a message - which doesn't always get to the care coordinator - and then wait. There is no way to directly contact them."

"I can't get hold of him. I call the number and leave a message, but he never calls me back."

"No direct line to the care coordinator. It takes a long time to get through and hear back-very frustrating."

"You have one [coordinator] for medical, but I don't have someone for everything else, like problems with my wheelchair or housing."

"No real understanding of client needs and choices."

\footnotetext{
${ }^{a}$ Number of individual survey respondents reporting a barrier within this category/theme.
}

the MCO won't change the pickup time because it is not three days notice." Finally, issues of trust arose when MCOs contracted with new, and often non-local, transportation providers.

\section{Communication}

Respondents cited various difficulties in communicating effectively with the MCOs. Fifty-one percent of survey 
respondents indicated they had contacted their MCO directly to ask any questions. Of those, $56.0 \%$ reported the MCO did not return their calls in a timely manner and $45.1 \%$ indicated that their questions were not answered satisfactorily.

Issues related to both accessibility and disability cultural competency were also raised. Several individuals with speech impairments reported that MCO staff did not take the time necessary to understand them. Another respondent stated, "The MCO said I could get a cell phone with 250 minutes for free, but as a quad [riplegic], I can't use their phone because the buttons are too small. I asked if I could use my existing phone with the same cell company, but was told they won't allow it." Consumers are also told to go online to get their questions answered, but respondents pointed out that not everyone has Internet access. Indeed, people with disabilities are significantly less likely to have access to and use the Internet than able-bodied individuals. ${ }^{27}$ Frequent and confusing mailings and requests for paperwork were also cited as problematic and overwhelming.

\section{Care coordination}

KanCare services for individuals on HCBS waivers include the provision of a Care Coordinator/Manager, a point person assigned and employed by the MCO who works directly with consumers to support them in managing their services. ${ }^{28}$ Only half of respondents knew who their Care Coordinator/Manager was. One consumer cited an inability to contact her Care Coordinator/Manager directly: "I can't get hold of him. I call the number and leave a message, but he never calls me back." In addition, respondents note that the coordination is medically oriented and fails to address non-medical concerns: "You have one [coordinator] for medical, but I don't have someone for everything else, like problems with my wheelchair or housing." Prior to KanCare, case managers at local agencies familiar with local resources had helped to address such concerns.

\section{Discussion}

The purpose of this study was to understand the experiences of Medicaid beneficiaries with disabilities who use LTSS as they moved to managed care in Kansas. Despite an overall satisfaction rating of $64 \%$, a number of important issues were raised related to provider networks, covered benefits, transportation, communications, and care coordination. Within these broad categories, lack of disability cultural competence and awareness of a range of accessibility issues were especially problematic.

In their study of transition to managed care for LTSS in three states, Saucier, et al $^{9}$ highlighted important considerations regarding continuity of care and provider choice. Similarly, Connolly and Paradise ${ }^{6}$ outlined issues of particular importance for people with disabilities, such as broad provider networks. Neither article documented the personal experiences of consumers. Our study of a small sample of people with disabilities moving to Medicaid managed care in Kansas provides real-time insights from beneficiaries during transition. They raise specific areas of concern that warrant further investigation and remedy by the MCOs to support enrollees in maintaining health and optimizing function.

Findings from this study suggest that, as managed care is extended into LTSS, MCOs will need to become more aware of, and responsive to, the needs of individuals with disabilities. Moreover, MCOs must be aware that nonmedical aspects of care may be equally important to the long-term health outcomes of this population as is more traditional medical care. Indeed, the National Council on Disability (NCD) ${ }^{14}$ developed 22 principles important in managed care systems for people with disabilities, many of which would have addressed issues identified by the Kansas beneficiaries. Moreover, $\mathrm{NCD}^{14}$ specifically recommended that "States planning to enroll Medicaid beneficiaries in managed long-term services should carefully analyze the diverse support needs among people targeted for enrollment and require managed care organizations (MCOs) to include skilled providers of such services and supports within their respective provider networks" (p. 20). As Connolly and Paradise ${ }^{6}$ suggest, and $\mathrm{NCD}^{14}$ affirms, disability-related measures of access and quality and rigorous monitoring are essential if people with disabilities are to have positive outcomes under managed care.

A few limitations of this study should be noted. First, the sample size is relatively small and data represent only one state's implementation of Medicaid managed care, thus limiting generalizability. The surveys were administered in the early months of KanCare implementation, and MCOs may currently be addressing some of the issues identified. Finally, because the purpose of the study was to identify problem areas needing attention, participants were asked to provide more information when difficulties or barriers were experienced, while positive experiences were not solicited as fully.

\section{Conclusion}

This study contributes seldom-considered first-person experiences among Medicaid beneficiaries with disabilities newly moved to Medicaid managed care. While not every participant identified problems, $45.7 \%$ of the sample reported problems in at least one of the five problem areas identified (Table 1), potentially representing thousands of individuals in the overall Kansas population using community-based LTSS. The results reported here provide specific examples of disability-related access and quality issues that must be addressed as more and more people with disabilities are placed into managed care arrangements for both their health services and their long-term services and supports. State policy makers and managed care organizations must be aware of these issues and address them 
proactively if managed care is to be effective for this population.

\section{Acknowledgments}

The authors would like to acknowledge their colleagues on this project from the University of Kansas Medical Center, Suzanne Hunt and Melissa Jaffee.

\section{References}

1. Iglehart J. Medicaid at a crossroads. N Engl J Med. 2011;364: $1585-1587$.

2. Kaiser Family Foundation (KFF). Medicaid Managed Care: Key Data, Trends and Issues. Publication 8046-02. Washington DC: Kaiser Family Foundation; 2012.

3. McCue M, Bailit M. Assessing the Financial Health of Medicaid Managed Care Plans and the Quality of Patient Care They Provide. Publication 1511-11. New York, NY: The Commonwealth Fund; 2011 Jun.

4. Burns M. Medicaid managed care and health care access for adult beneficiaries with disabilities. Health Serv Res. 2009;44(5 Pt 1): $1521-1541$.

5. Graham C, Kurtovich E, Taube S. Transitioning Beneficiaries with Complex Care Needs to Medicaid Managed Care: Insights from California. Publication 8453. Washington DC: Kaiser Family Foundation; 2013 Jun.

6. Connolly J, Paradise J. People with Disabilities and Medicaid Managed Care: Key Issues to Consider. Publication 8278. Washington DC: Kaiser Family Foundation; 2012 Feb.

7. Summers L. Medicaid Long-term Services and Supports: Key Considerations for Successful Transitions from Fee-for-service to Capitated Managed Care Programs. Publication 8433. Washington DC: Kaiser Family Foundation; 2013 Apr.

8. Kaiser Family Foundation (KFF). Medicaid Moving Forward (June 2014 Factsheet). Washington DC: Kaiser Family Foundation; 2014.

9. Saucier P, Burwell B. Gold. The Growth of Managed Long-term Services and Supports (MLTSS) Programs: A 2012 Update. Ann Arbor, MI: Truven Health Analytics; 2012.

10. Saucier P, Burwell B, Halperin A. Consumer Choices and Continuity of Care in Managed Long-term Services and Supports (MLTSS): Emerging Practices and Lessons. Washington DC: American Association of Retired Persons; 2013.

11. Kansas Medicaid KanCare Web site. What is KanCare? Available at: http://kancare.ks.gov/whats_kancare.htm; Accessed 26.06.14.
12. Iezzoni L. Imperatives for HSR addressing: individuals with disabilities - the canaries in health care's coal mine. Med Care. 2013;51: $133-136$.

13. Medicaid and CHIP Payment and Access Commission (MACPAC). Report to Congress on Medicaid and CHIP. Washington DC: MACPAC; 2013.

14. National Council on Disability (NCD). Medicaid Managed Care for People with Disabilities: Policy and Implementation Considerations for State and Federal Policymakers. Washington DC: NCD; 2013.

15. Palmer A, Howell E, Costich J, et al. Evaluation of Statewide Risk-based Managed Care in Kentucky: A First Year Implementation Report. Washington DC: The Urban Institute; 2012.

16. Hall J, Kurth N, Hunt S. Employment as a health determinant for working-age, dually-eligible people with disabilities. Disabil Health J. 2013:6:100-106.

17. Lincoln Y, Guba E. Naturalistic Inquiry. Newbury Park, CA: Sage; 1985.

18. Hsieh H-F, Shannon SE. Three approaches to qualitative content analysis. Qual Health Res. 2005;15:1277-1288.

19. Emerson RM, Fretz RI, Shaw LL. Writing Ethnographic Fieldnotes. 2nd ed. Chicago, IL: University of Chicago Press; 2011.

20. Hughes C, Gooze R, Finkelstein D, Whitaker R. Barriers to obesity prevention in head Start. Health Aff. 2010;29(3):454-462.

21. Dupree JM, Patel K, Singer SJ, et al. Attention to surgeons and surgical care is largely missing from early Medicare Accountable Care Organizations. Health Aff. 2014;33(6):972-979.

22. Vuckovic N. Self-care among the uninsured: 'you do what you can do'. Health Aff. 2000;19(4):197-199.

23. Choi K, Fabian L, Mottey N, Corbett A, Forster J. Young adults' favorable perceptions of snus, dissolvable tobacco products, and electronic cigarettes: findings from a focus group study. Am J Public Health. 2012;102(11):2088-2093.

24. Hall JP, Carroll S, Moore JM. Health care behaviors and decisionmaking among enrollees in a state high risk insurance pool: focus group findings. Am J Health Promot. 2010;24(5):304-310.

25. Henrich NJ, Dodek P, Heyland D, et al. Qualitative analysis of an intensive care unit family satisfaction survey. Crit Care Med. 2011;39(5):1000-1005.

26. Bruffet K. Home and Community Based Services (HCBS) Program Update. Presented at: Meeting of the Kansas Department for Aging and Disability Services Robert G Bethell Joint Committee on Home and Community Based Services and KanCare Oversight; August 12, 2014. Topeka, KS.

27. Fox S. Americans Living with Disability and Their Technology Profile. Washington DC: Pew Research Center; 2011.

28. Kansas Medicaid KanCare Web site. Consumer frequently asked questions. Available at: http://www.kancare.ks.gov/consumer_faqs.htm; Accessed 26.06.14. 